\title{
针刺与 TDP 理疗结合拔罐治疗慢性盆腔炎的临床应用效果观察
}

\section{Clinical Application Effect Observation of Acupuncture and TDP Physiotherapy Combined with} Cupping in the Treatment of Chronic Pelvic Inflammation 王少河

\author{
Shaohe Wang
}

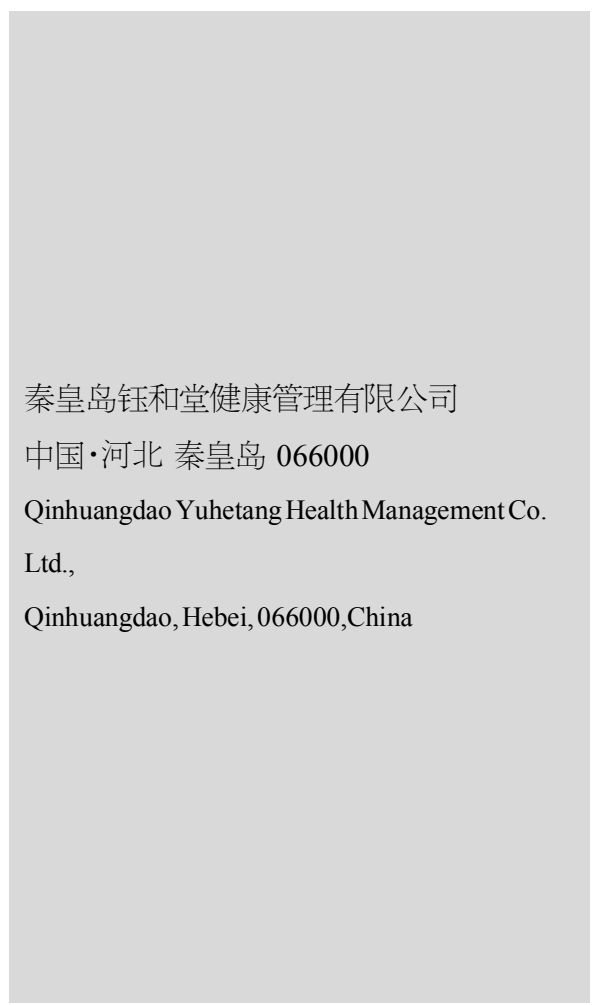

\section{1 资料和方法}

\section{1 一般资料}

选取我院 2018 年 12 月至 2019 年 11 月收治的 100 例慢 性盆腔炎患者作为研究对象,所有患者均符合《妇产科学》中 关于慢性盆腔炎疾病的相关诊断标准，治疗方法均由患者自 行选择, 本院于患者选择前对两种治疗方法流程均进行详细 说明。

纳入标准: 所有患者均对此次研究知情,并签署知情同意 书。

排除标准: 排除存在沟通功能障碍或精神疾病患者; 排除 不遵守医嘱按时进行治疗的患者。

对照组 50 例, 年龄 28 48 岁, 平均年龄 $(38.2 \pm 0.4)$ 岁, 病 程 1 8 年, 平均病程 $(4.5 \pm 0.4)$ 年。观察组 50 例, 年龄 $27 \sim 49$ 岁, 平均年龄 $(38.4 \pm 0.3)$ 岁, 病程 $2 \sim 7$ 年, 平均病程 $(4.5 \pm 0.2)$ 年。两组患者在年龄、病程方面对比无较大差异 $(P>0.05)$, 具有 良好的可比性。

\section{2 方法}

对照组采取常规微波照射治疗，具体治疗方法为使用微 波多功能治疗仪对患者下腹痛的压痛点进行照射治疗, 每次 照射时间为 20 分钟,每日照射一次, 持续照射一个疗程即 12 天,可于经期前后进行,避免因经期中断治疗。持续照射三个 疗程,一月一个疗程, 可以经期为分界点, 三个疗程结束后, 观 察治疗效果 [1]。

观察组采取针刺与 TDP 理疗结合拔罐治疗, 具体治疗方 法为: 首先为患者进行取穴工作, 取患者合谷、关元、水道、中 极、归来、足三里、三阴交、阴陵泉穴位, 使用 30 号 1.5 寸毫针, 让患者排空小便后保持平躺, 并对患者的穴位进行常规消毒 工作。消毒完成后便可行针，向患者关元、中极两穴位直刺 $0.8 \sim 1.0$ 寸, 并保持针感要求达到患者阴部; 向患者水道、归来 两穴位直刺 $0.8 \sim 1.2$ 寸, 并保持针感向附件部放射。行针时保 持手法提插轻匀, 并进行左右的小幅度捻转, 结束后留针 30

(下转第 28 页) 
临床医学研究 Clinical Medicine Research

以百分比表示, 采用 $\chi^{2}$ 检验, 以 $P<0.05$ 为差异有统计学意义。

\section{2 结果}

\section{1 观察组与对照组患者护理前后 SAS、SDS 评分对比}

观察组与对照组患者护理前后 SAS、SDS 评分具体如表 1 所示:

表 1 观察组与对照组患者护理前后 SAS、SDS 评分对比

\begin{tabular}{|c|c|c|c|c|}
\hline \multirow{2}{*}{ 组别 } & \multicolumn{2}{|c|}{ SAS } & \multicolumn{2}{|c|}{ SDS } \\
\hline & 护理前 & 护理后 & 护理前 & 护理后 \\
\hline 观察组 & $37.56 \pm 8.22$ & $22.53 \pm 4.95$ & $37.64 \pm 7.86$ & $20.02 \pm 3.52$ \\
\hline 对照组 & $37.11 \pm 7.95$ & $29.69 \pm 5.68$ & $38.96 \pm 5.24$ & $28.54 \pm 5.18$ \\
\hline$P$ & 0.512 & 5.698 & 0.538 & 6.253 \\
\hline$t$ & 0.623 & 0.001 & 0.436 & 0.002 \\
\hline
\end{tabular}

\section{2 观察组与对照组患者预后情况对比}

观察组与对照组患者预后情况具体如表 2 所示:

表 2 观察组与对照组患者预后情况对比

\begin{tabular}{c|c|c|c|c}
\multicolumn{5}{c}{} \\
\hline 组别 & 心肌梗死 & 肝功能异常 & 血管重建 & 胃肠道反应 \\
\hline 观察组 & 0 & 1 & 1 & 2 \\
\hline 对照组 & 4 & 4 & 3 & 5 \\
\hline$\chi^{2}$ & 3.246 & 5.616 & 5.133 & 5.663 \\
\hline$P$ & 0.000 & 0.002 & 0.001 & 0.026 \\
\hline
\end{tabular}

\section{( 上接第 26 页)}

分钟。同时, 以 TDP 理疗患者的下腹部, 时间也为 30 分钟, 并 保持 $0.3 \sim 0.4 \mathrm{~m}$ 的距离。最后, 在针刺、TDP 理疗结束后, 在患者 肾俞、第十七椎穴、八髎位置进行拔罐治疗, 时间为 10 15 分 钟。每日治疗一次,一月治疗十次,每两次间隔一日,可于经期 后进行, 避免因经期中断。十次为一疗程, 连续治疗三疗程, 三 疗程后, 观察治疗效果。

\section{3 评判标准}

对比两组患者的治疗效果。治疗效果分显著、有效、一般 三种标准。治疗效果显著, 表现为治疗结束后患者的身体完全 康复, 不适症状尽数消失或医疗检测中患者的病灶部位恢复 正常; 治疗效果有效, 表现为治疗结束后患者身体的不适症状 好转显著或医疗检测中患者的病灶部位状况明显改善; 治疗 效果一般, 表现为治疗结束后患者的身体状况无明显变化或 医疗检测中患者的病灶部位无明显变化。另外, 治疗总有效 率=显著+有效。

\section{4 统计学处理}

本研究数据均使用 SPSS22.0 系统进行处理, 计量资料以 均数 \pm 标准差 $(\bar{x} \pm \mathrm{s})$ 表示, 采用 $t$ 检验, 计数资料以百分比表 示, 采用 $\chi^{2}$ 检验, $P<0.05$ 为差异有统计学意义。

\section{3 讨论}

医疗技术水平的提升降低了急性冠脉综合征的死亡概 率,但患者的病情愈后情况有待加强。该病愈后对患者的生活 质量有了全新的要求，因此在急性冠脉综合征患者的护理过 程中, 医护人员应对其进行有针对性的心理疏导。共情护理能 够有效提升急性冠脉综合征患者的护理有效率。根据此次分 组研究结果可知, 护理后, 观察组患者的 SAS、SDS 评分显著 优于对照组患者, $P<0.05$; 观察组患者心肌梗死、肝功能异常、 血管重建、胃肠道反应的情况均优于对照组患者, $P<0.05$ 。

\section{4 结语}

综上所述，医护人员给予急性冠脉综合征患者换位思考 联合共情护理, 对其心理状态及病情预后有十分积极的影响, 而且能够改善患者的生活质量, 对病情的恢复有促进作用, 该 护理方式的护理疗效极为显著,应推广使用。

\section{参考文献}

[1]冒云霞,李英,黄荔荔.换位思考联合共情护理对急性冠脉综合 征患者心理状态及预后的影响[J].齐鲁护理杂志,2019,25(19):82-84.

[2]朱萍,杨承健.双心护理联合放松训练对急性冠脉综合征患者 心理状态的影响[J].护理学杂志,2017,32(21):89-90+96.

\section{2 结果}

观察组的治疗总有效率显著高于对照组 $(P<0.05)$, 详情 见表 1 。

表 1 两组患者治疗效果对比

\begin{tabular}{c|c|c|c|c}
\hline 组别 & 显著/例 & 有效/例 & 一般/例 & 总有效率/\% \\
\hline 对照组 & 10 & 24 & 16 & 68.0 \\
\hline 观察组 & 20 & 24 & 6 & 88.0 \\
\hline$\chi^{2}$ & 4.762 & 0.000 & 5.828 & 5.828 \\
\hline$P$ & 0.029 & 1 & 0.016 & 0.016 \\
\hline
\end{tabular}

\section{3 结语}

中医学认为, 女性乃热盛阳六之体, 在外来因素的影响 下, 气血容易亏虚, 湿热毒邪乘机侵入体内, 与人体血互结然 后下注, 从而损伤人体带脉, 进而引发慢性盆腔炎。

在本研究中，观察组患者的治疗总有效率显著高于对照 组 $(P<0.05)$ 。由此表明, 在慢性盆腔炎患者治疗中采取针刺与 TDP 理疗结合拔罐治疗可以起到良好的治疗效果, 促进患者 的身体康复。

\section{参考文献}

[1]李艺. 慢性盆腔炎妇产科临床治疗效果观察[J].当代临床医刊, 2016,29(5):2572. 